Health and Medicine | Professor Allan Hackshaw

\section{No safe level of smoking exists for heart disease and stroke}

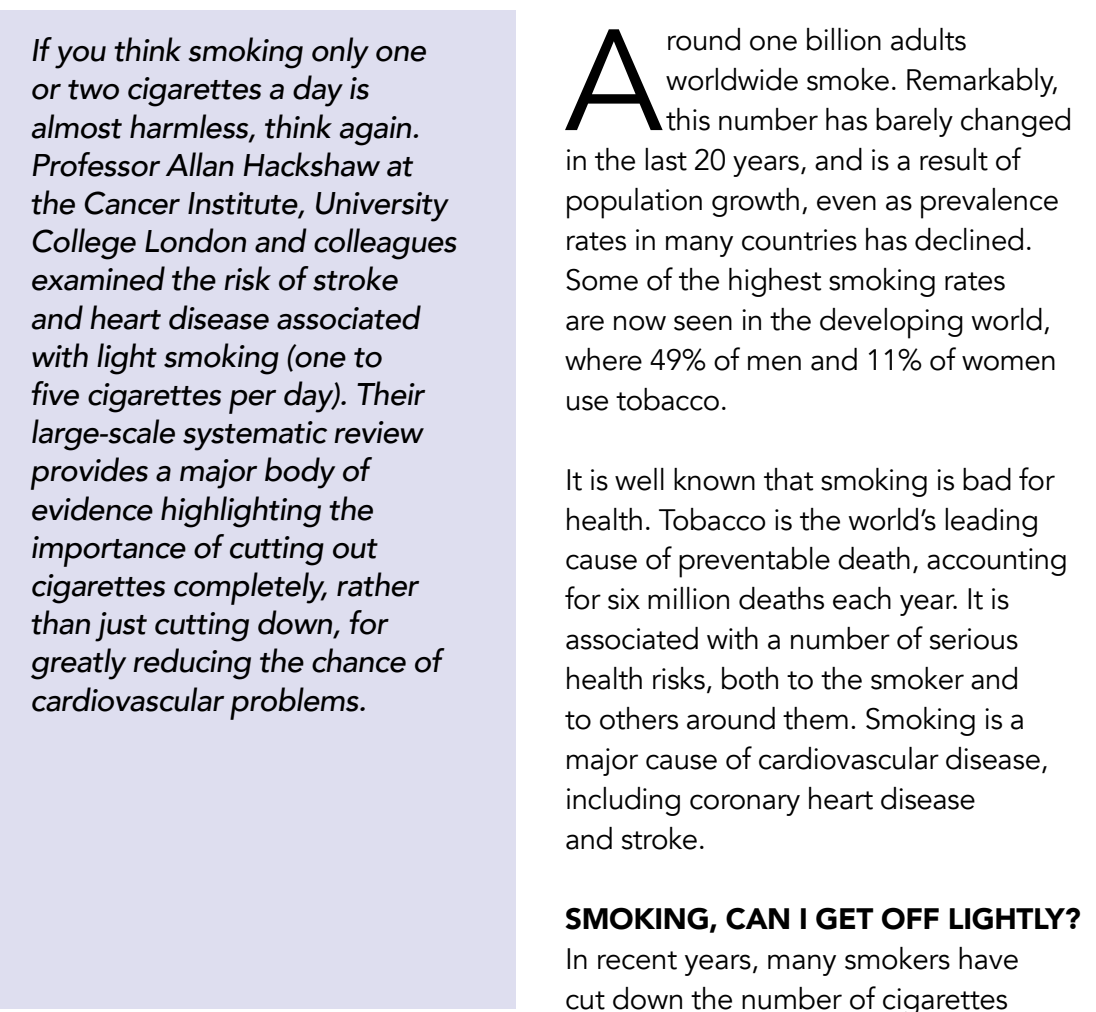

hat they smoke, rather than giving up completely. A recent Health Survey for England reported that $26 \%$ of current smokers stated that they wanted to not trying to stop, and $40 \%$ stated that they smoked less than in the previous year. The percentage of smokers who consume one to five cigarettes per day has steadily increased over the last decade, both in the UK and USA. This is partly due to the common belief that smoking only one or two cigarettes a day is relatively safe. There is an intuitive assumption that by reducing consumption you could expect to reduce risk in a proportionate way. For example, reducing the number of cigarettes from 20 to one per day, you might assume that this has $1 / 20$ th $(5 \%)$ the amount of the extra risk that is due to smoking. Indeed, his assumption seens to hold true for several types of cancer. disease and stroke?

Tobacco is the world's leading cause of preventable death, accounting for six million deaths each year.

6

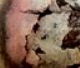

SMOKING: RISKS BROUGHT

Professor Allan Hackshaw at University College London (UCL) and colleagues the assumption that smoking fewer cigarettes has a much lower risk of heart disease or stroke. They directly investigated the effects of light smoking through a large meta-analysis of observational studies, enabling them to quantify the risk of coronary heart disease and stroke.

The team conducted a largescale review of 55 medical journal publications, combining the evidence from 141 cohort studies of heart diseas and stroke in smokers and never(n) people in total. Examining the risk of moking one, five or 20 cigarettes per who had never smoked. The results were then pooled and examined to see how light smoking (defined as on or five cigarettes each day) affected health, compared to heavy smoking (20 cigarettes each day). The researchers also allowed for potential confounding factors (in addition to age and sex) such as body mass index, blood pressure and e British Medical Journal.

Notably, the researchers showed that the impact of light smoking on heart expected Smoking just one cigarette day accounted for half of the excess isk of cardiovasular disease associated with smoking 20 a day in men, and for one third of the excess risk in women. Specifically, they found that men who smoke about one cigarette per day have a $48 \%$ (all studies) to $74 \%$ (studies controlling for confounders) higher risk of heart disease than men who never smoked, and a $25 \%$ higher risk of stroke.

\section{WOMEN AT HIGHER RISK}

The estimates were found to be higher in women who smoked between one and five cigarettes per day: $5 \%$ (all studies) to $11 \% \%$ (studies controlling for confound ars) $31 \%$ for stroke, cont disease, and $31 \%$ for stroke, compared day, they compared findings to those cholesterol levels, that may have skewed results. Their findings were published in

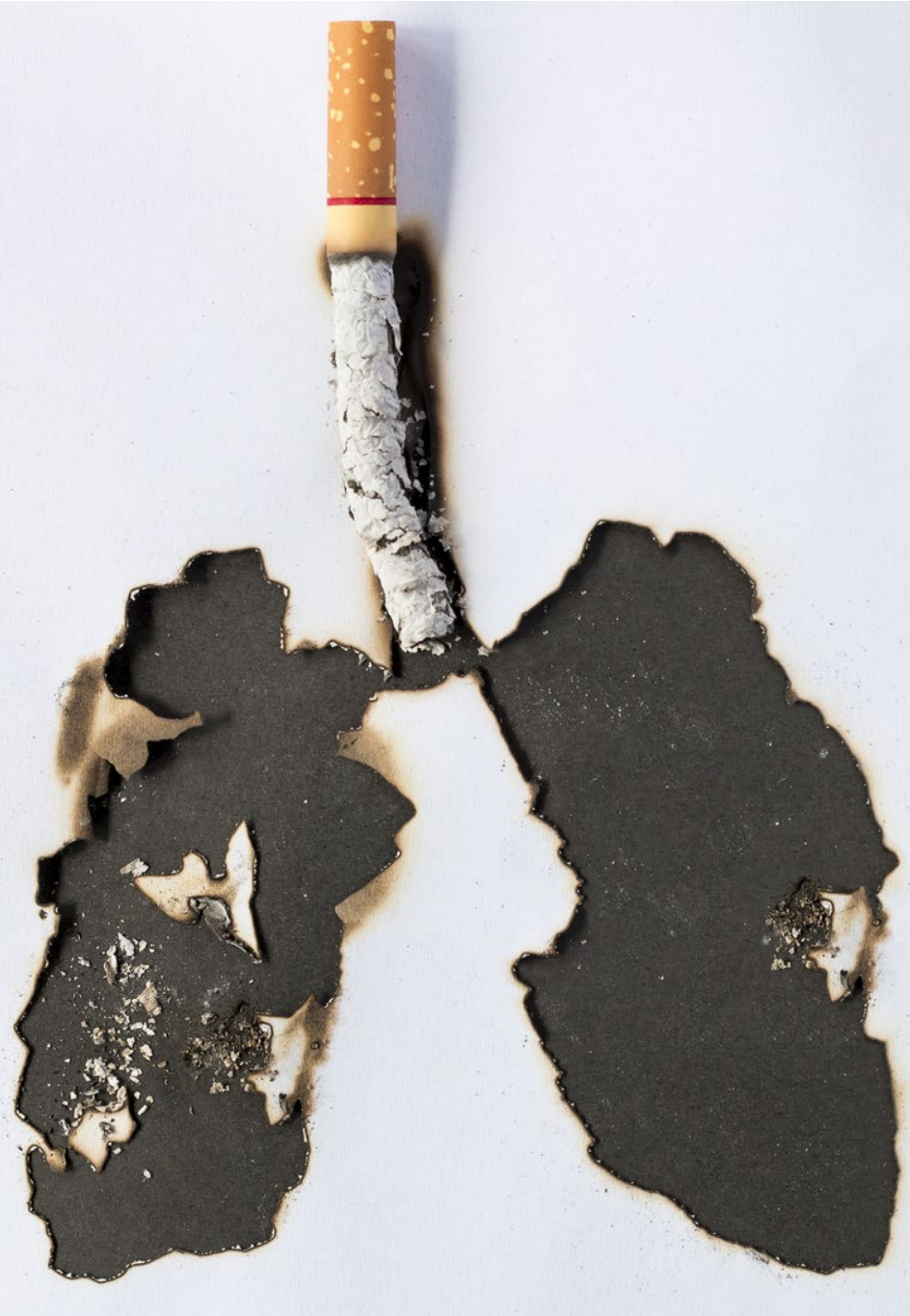

Smoking just one cigarette a day accounts for half of the extra risk of heart disease and stroke associated with smoking 20 a day in men, and for one third of the extra risk in women.

evidence is in line with other studies
that suggest that the harmful effects of smoking are greater in women than in men.

\section{MPROVING KNOWLEDGE} ABOUT SMOKING

The BMJ paper is the first of its kind to combine results across so many

This well-conducted study demonstrates that there is no safe level of smoking. Professor Hackshaw confirms. "We show that a large proportion of the risk of is due to tobacco comes from smoking only a few cigarettes. This has important almost harmless." roke. It is an important providers alike. It also represents a public health campaigns and smoking provide a stronger and positive should provide a stronger and positive incentive rather than cut down. coronary heart disease and stroke that publich 


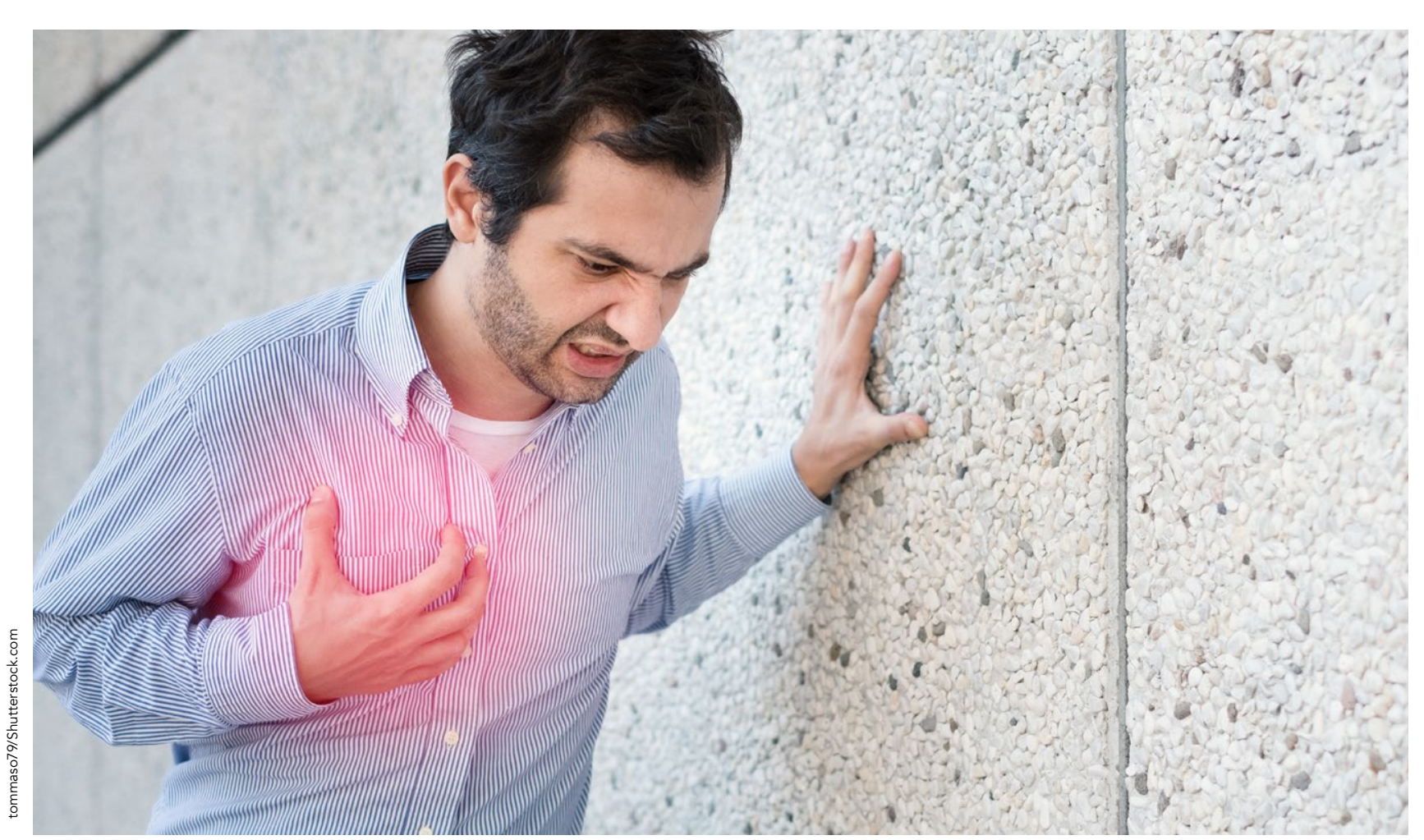

It is incorrect to assume smoking only a couple of cigarettes each day carries little or no harm.

The BMJ study goes against what many people believe - that smoking a lot less means proportionately lower risks to health. Professor Hackshaw says: "Smokers who cut down the number of cigarettes they use can benefit from large reductions in the risk of cancer, as well as some benefits on cardiovascular disease. However, smoking only one

with a risk of coronary heart disease and stroke that is still substantially higher than many smokers or health professionals recognise (as much as haf the excess risk of smoking 20 per day STUBBING OUT CIGARETTE USE Coronary heart disease and stroke are with 370,000 and 140,000 deaths foa

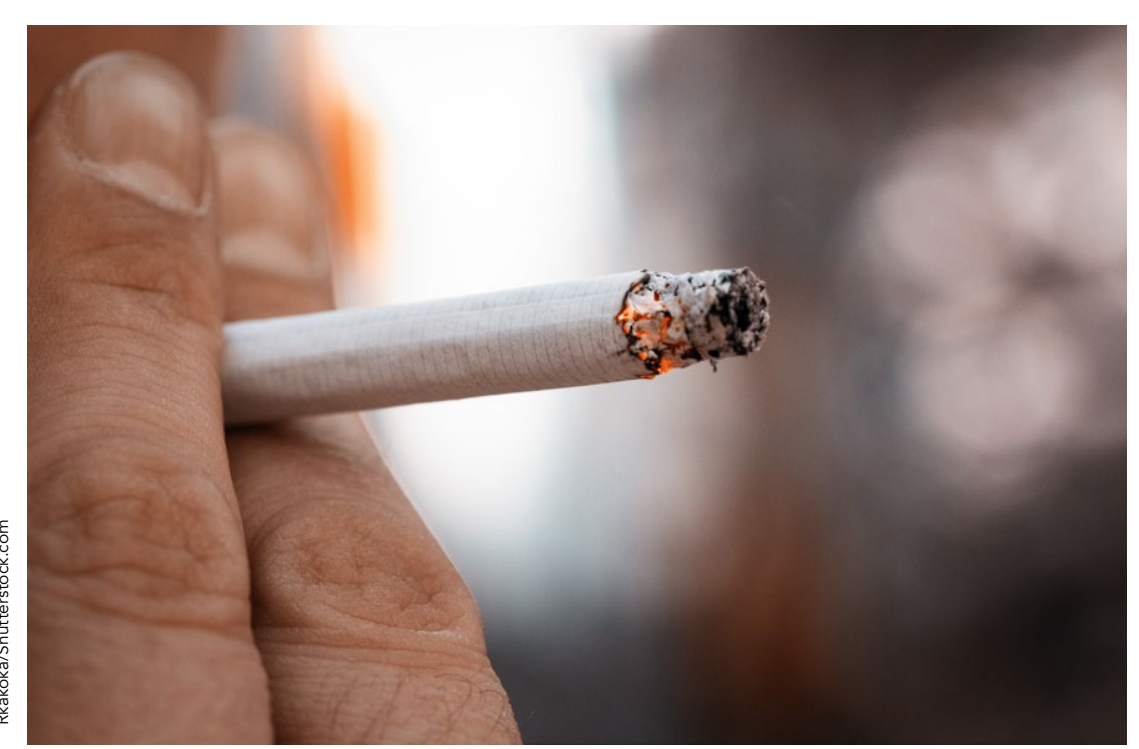

heart disease and stroke, respectively each year in the US alone (compared with 155,000 for lung cancer). About 20\% of all cardiovascular disease is thought number of deaths, however, is eclipsed by the number of episodes, meaning that many more people are living with cardiovascular disease. Not only does this have a major impact on their menta social and physical well-being, it also places a large burden on health services.

Cutting out cigarettes completely has huge benefits. Importantly, the risk of carcilovascular disease is greatly reduced interest and discussion is the methods available to smokers to help them quit Electronic cigarettes have received a lot of attention. Although not thought to be completely harmless, e-cigarettes are believed to be much safer than cigarettes. Until more studies examining the effects of e-cigarettes are complete, a key consideration is the balance of harms. Is it better to reduce the number of cigarettes smoked down to one to five per day, or to stop completely by switching to e-cigarettes? The evidence the BMJ paper shows substant disease for light smoking which are to outweigh any harms that might be due
to e-cigarettes. disease for light smoking, which are likely

\section{[5 \\ Behind the Research}

I.

\section{Professor Allan Hackshaw}

E: a hackshaw@ucl.ac.uk T: $+4402076799893 \quad$ W: http://www.ctc.uclacuk

\section{Research Objectives}

Professor Hackshaw's research involves the design, analysis and conduct of

\section{Detail}

Professor Allan Hackshaw

Cancer Research UK \& UCL Cancer Trials Centre Cancer Institute

OOT

ondon W1T CT

Bio

Professor Hackshaw is Professor of Epidemiology \& Medical Statistics at University College London, and previously at the Wolfson Institute for Preventive Medicine. A key area of his work is tobacco and health, including passive smoking and smoking during pregnancy. He has written major journal articles and book chapters in this field.

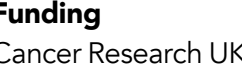

Collaborators

作 - Sadie Boniface (Kings College London)

Duša Mil ković(Univariy College London/Evider

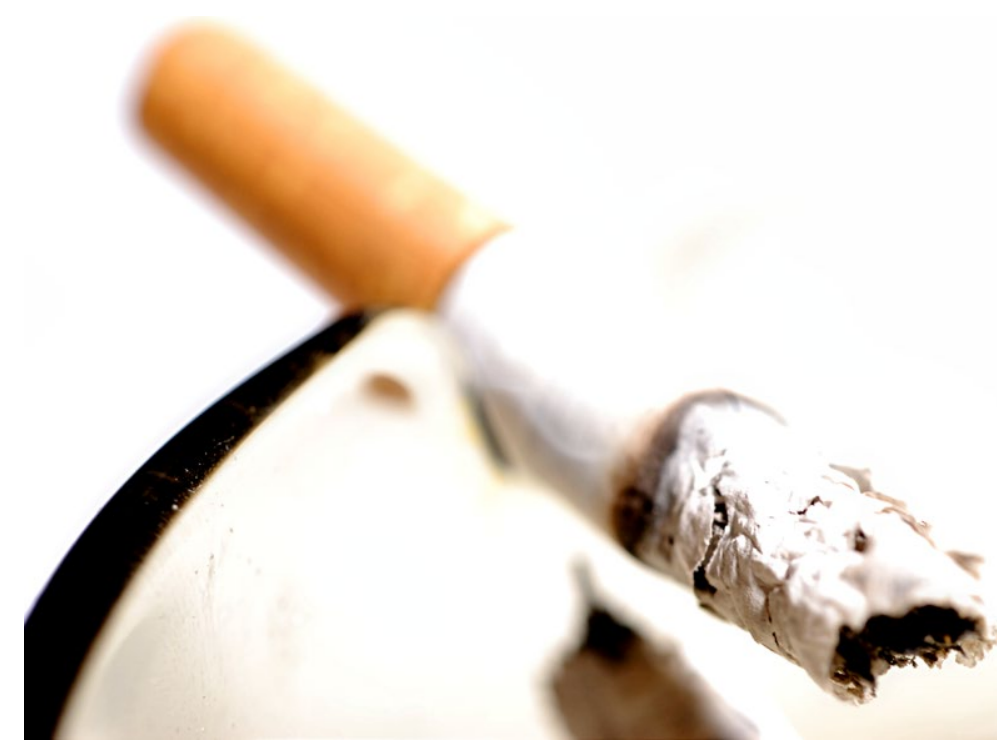

References

Allan Hackshaw, Joan K Morris, Sadie Boniface, Jin-Ling Tan Dušan Milenkovic. (2018). Low cigarette consumption and

isk of coronary heart distease and stroke. meta-analysis of

141 cohort studies in 55 study reports. BMJ 360:55855. doi 10.1136/bmj.j5855

\section{Personal Response}

Your BMJ study gives convincing evidence that light smoking carries higher risks for cardiovascular dise than for certain smoking-related cancers. Can you explain why the reduction of risk is different for cancer?

II The effects of smoking on cancer usually take several or many years to be seen, arising through a more gradua
build-up of cells that have abnormalities which then turn into cancer. There seems to be a near-linear relationship between how much people smoke and cancer risk. However, he cardiovascular system is particularly sensitive much quicker adverse impact on heart disease and stroke, typically taking only a few years to be seen, and we can see biological changes even with only a little amount

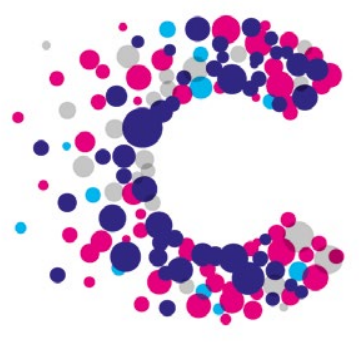

CANCER RESEARCH UK

Cancer Research UK and ${ }^{\bullet} \bullet$ UCL Cancer Trials Centre

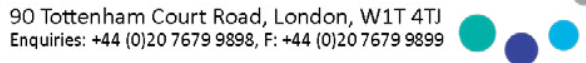

